\title{
Constraints for the aperiodic O-mode streaming instability
}

\author{
M. Lazar ${ }^{1,2}$, R. Schlickeiser ${ }^{2,3}$, S. Poedts ${ }^{1}$, A. Stockem ${ }^{2}$ and S. Vafin ${ }^{2 *}$ \\ 1 Center for Plasma Astrophysics, Celestijnenlaan 200B, 3001 Leuven, Belgium \\ 2 Institut für Theoretische Physik, Lehrstuhl IV: Weltraum- und Astrophysik, \\ Ruhr-Universität Bochum, D-44780 Bochum, Germany \\ 3 Research Department Plasmas with Complex Interactions, \\ Ruhr-Universität Bochum, D-44780 Bochum, Germany
}

(Dated: December 19, 2014)

\begin{abstract}
In plasmas where the thermal energy density exceeds the magnetic energy density $\left(\beta_{\|}>1\right)$, the aperiodic ordinary mode (O-mode) instability is driven by an excess of parallel temperature $A=T_{\perp} / T_{\|}<1$ (where $\|$ and $\perp$ denote directions relative to the uniform magnetic field). When stimulated by parallel plasma streams the instability conditions extend to low beta states, i.e., $\beta_{\|}<1$, and recent studies have proven the existence of a new regime, where the anisotropy threshold decreases steeply with lowering $\beta_{\|} \rightarrow 0$ if the streaming velocity is sufficiently high. However, the occurrence of this instability is questionable especially in the low-beta plasmas, where the electrostatic two-stream instabilities are expected to develop much faster in the process of relaxation of the counterstreams. It is therefore proposed here to identify the instability conditions for the O-mode below those required for the onset of the electrostatic instability. An hierarchy of these two instabilities is established for both the low $\beta_{\|}<1$ and large $\beta_{\|}>1$ plasmas. The conditions where the O-mode instability can operate efficiently are markedly constrained by the electrostatic instabilities especially in the low-beta plasmas.
\end{abstract}

PACS numbers: 52.25.-b - 52.25.Mq - 52.25.Xz - 52.35.Fp

Keywords: magnetized plasma - electromagnetic instabilities - counterstreams - temperature anisotropy space plasmas

\section{INTRODUCTION}

There is an increased interest for understanding the mechanisms that can destabilize the aperiodic or weakly propagating modes in anisotropic plasmas. Of these the purely growing (aperiodic, i.e., $\Re(\omega)=0$ ) ordinary $(\mathrm{O})$ mode instability has recently received particular attention owing to its potential applications in space plasmas $^{1-4}$. In a plasma at rest the O-mode instability (OMI) can develop only if the plasma beta is sufficiently high $\left(\beta \equiv 8 \pi n k_{B} T / B_{0}^{2}>2\right)$, and thermal energy is higher in the direction parallel to the uniform magnetic field ( $\left.\mathbf{B}_{0}\right)$, i.e., $A=T_{\perp} / T_{\|}<1^{5-8}$.

In the presence of streams, propagating along the ordered magnetic field, the activity of this instability extends to low beta $\beta<1$ states $^{2-4,9-11}$ (although the instability is inhibited by the magnetic field by limiting the range of unstable wavenumbers ${ }^{1}$ ). Thus, for two symmetric counterstreams of electrons (subscript $e$ ) the conditions necessary for the O-mode instability are ${ }^{9}$ $\beta_{e, \|}>2 /\left(1+2 V_{e}^{2} / u_{e, \|}^{2}\right)$ and $A_{e}<1+2 V_{e}^{2} / u_{e, \|}^{2}$, where $u_{e, \|}=\left(2 k_{B} T_{e, \|} / m_{e}\right)^{1 / 2}$ is the electron thermal velocity in parallel direction, $\beta_{e, \|}=8 \pi n k_{B} T_{e, \|} / B_{0}^{2}$, and $V_{e}$ is the streaming velocity. (Symmetric counterstreams enable to analyze the O-mode decoupled from the extraordinary (X) mode, which is less susceptible to the instability. Fig. 1 presents a schematic with the possible configu-

*Electronic address: mlazar@tp4.rub.de rations of symmetric counterstreams.) These conditions simply show that instability is also predicted for low values of $\beta_{e, \|}<1$ (if streaming velocity is large enough $V_{e}>u_{e, \|} / \sqrt{2}$ ), and for high values of the anisotropy $A_{e}>1$ (given by an excess of perpendicular temperature). Growth rates are of the order of electron cyclotron frequency $\left|\Omega_{e}\right|$.

A plasma system with counterstreaming ions can be more susceptible to the O-mode instability than one in which only electrons are streaming ${ }^{10,11}$. The streaming ions enlarge the range of unstable wavenumbers but affect only slightly the maximum growth rates. However, for large growth rates (of the order of $\left|\Omega_{e}\right|$ ), high electron beta $\beta_{e, \|} \sim 1$ and temperature anisotropy in parallel direction, i.e., $T_{e, \|} \gg T_{e, \perp}$, are needed. On the other hand, only sufficiently high streaming velocities $V_{e}>u_{e, \|}$ can predict the occurrence of instability at large $A_{e}>1$.

Recently, a number of studies have been devoted to express analytically the marginal condition of the O-mode instability $^{2-5}$. It is now straightforward to determine the instability conditions for the whole range of plasma beta, including the low-beta regime where the O-mode is driven unstable only by the relative motion of the plasma streams ${ }^{2,4}$. For low $\beta_{\|}<1$ it is shown for the first time the existence of a new regime, where the anisotropy thresholds steeply decrease with lowering $\beta_{\|} \rightarrow 0$ if the streaming velocity is sufficiently high. The exact instability thresholds have been derived numerically for small but finite growth rates ${ }^{3}$, in order to confirm the marginal instability condition in analytical forms, and implicitly the existence of the new regime in the low-beta limit.

Counterstreaming plasmas are also subject to the elec- 
FIG. 1: Schematic of three possible cases of symmetric counterstreams: I. Electrons and ions have the same streaming velocity $V_{e}=V_{i}$; II. The electron streaming velocity is higher than the ion streaming velocity $V_{e}>V_{i}$; III. Ions are stationary $V_{i}=0$.

trostatic two-stream instabilities (TSI), e.g., electronelectron, electron-ion and ion-ion, of which, the instabilities driven by electrons (with a growth rate of the order of electron plasma frequency $\omega_{p e}=\sqrt{4 \pi n e^{2} / m_{e}}$ ) appear to be faster ${ }^{12,13}$. Moreover, the electrostatic twostream instability is in most scenarios faster than the O-mode instability (OMI), except for streaming velocities very near or below the threshold for the onset of the two-stream instability ${ }^{10,11}$. The instability of the Omode remains to be established only for streaming velocities below the threshold of the two-stream instability even for low $\beta_{e, \|}<1$. In this paper we propose to delimitate these regimes on the basis of the results in Ref. 13, where the marginal condition has been derived systematically for different types and characteristics of two-stream instabilities. Thus, in Sec. II we revisit the instability conditions of the O-mode for three specific cases of symmetric counterstreams. These are confronted in Sec. III with the instability conditions of the electrostatic twostream modes, providing the existence conditions for the O-mode streaming instability. The new criteria are discussed along with our final conclusions in the last section.

\section{THE O-MODE INSTABILITY}

We first reanalyze the O-mode instability invoking the recent results in Refs. 2 and 4 . These results are here applied for three specific cases of symmetric counterstreams with each component modeled by a drifting biMaxwellian distribution function. A schematic of these plasma systems is presented in Fig. 1: I. The streams are neutral with electrons and ions having the same streaming velocity $V_{e}=V_{i}$; II. The electron streaming velocity is higher than the ion streaming velocity, $V_{e}>V_{i}$; and III. Ions are at rest, $V_{i}=0$, and only electrons are streaming. Symmetric counterstreams minimize the number of employed parameters, and enable to analyze the O-mode decoupled from the extraordinary (X-) mode, which is less susceptible to being unstable. Only counterstreams of the same species need to be symmetric to satisfy this condition, but the electron and ion properties (e.g., streaming velocity, parallel or perpendicular temperature) are not necessary the same. We are dealing with a single species of ions, namely, protons.

\section{A. Preliminary conditions: general case}

We assume two-component streams of electron-proton plasmas, and start their stability analysis from the analytical Eq. (49) in Ref. 4

$$
\begin{aligned}
& \mu^{2} y^{2}+\left(1+\mu^{2}\right)\left[1-\frac{\beta_{\|}}{2}-P_{e} \frac{\mu^{2}}{1+\mu^{2}}\left(1+\frac{1}{\nu \mu}\right)\right] y \\
& =\beta_{\|}-1\left(1+\frac{\mu}{\nu}\right) P_{e}
\end{aligned}
$$

with the same streaming parameters

$$
\nu \equiv \frac{\epsilon_{e 1}}{\epsilon_{p 1}} \frac{1-\epsilon_{p 1}}{1-\epsilon_{e 1}} \frac{V_{e 1}^{2}}{V_{p 1}^{2}}, \quad P_{e} \equiv \frac{4 \pi m_{e} n_{0} V_{e 1}^{2}}{B_{0}^{2}} \frac{\epsilon_{e 1}}{1-\epsilon_{e 1}},
$$

introduced in Ref. 4, and $y^{1 / 2} \equiv x_{0}$ being the limit value of the squared normalized wavenumber $x_{e} \equiv k^{2} u_{\perp, e}^{2} /\left(2 \Omega_{e}^{2}\right)=k^{2} c^{2} A_{e} \beta_{\|} /\left(2 \omega_{p e}^{2}\right)$ required by the marginal condition of instability $(\Im(\omega) \equiv \gamma=0)$, This equation is obtained based on the improved approximations (36) and (37) in the same reference, and here in the next will be refined by neglecting $1 \ll \mu^{2}$ (or $\mu^{-2} \ll 1$ ), where $\mu=m_{p} / m_{e}=1836$ is the proton-electron mass ratio, and removing the restriction to very high values of the parameter $\nu$. Because of the symmetry of the counterstreams of each species (with the same relative density $\epsilon_{e 1}=\epsilon_{e 2}=1 / 2, \epsilon_{p 1}=\epsilon_{p 2}=1 / 2$, and the same streaming velocity $\left.V_{e 1}=V_{e 2}=V_{e}, V_{p 1}=V_{p 2}=V_{p}\right)$ the quantities $\nu$ and $P_{e}$ simplify as follows

$$
\nu=\frac{V_{e}^{2}}{V_{p}^{2}}, \quad P_{e}=\frac{\omega_{p e}^{2}}{\Omega_{e}^{2}} \frac{V_{e}^{2}}{c^{2}} .
$$

Also for simplicity, the electron and ion temperatures are assumed equal $\left(T_{e, \|}=T_{p, \|}=T_{\|}, T_{e, \perp}=T_{p, \perp}=T_{\perp}\right)$, implying $\beta_{e, \|}=\beta_{p, \|}=\beta_{\|}$.

Equation (1) then reads

$$
y^{2}+a y+b=0
$$

with

$$
\begin{gathered}
a=1-\frac{\beta_{\|}}{2}-P_{e}\left(1+\frac{1}{\nu \mu}\right), \\
b=\frac{1}{\mu^{2}}\left[1-\beta_{\|}-\left(1+\frac{\mu}{\nu}\right) P_{e}\right],
\end{gathered}
$$

This equation admits a positive solution $y>0$ when at least one of the two coefficients $a$ or $b$ is negative. In a low $\beta<1$ regime this condition is satisfied when the terms depending on $P_{e}$ are large enough. Thus, $a<0$ is satisfied if

$$
P_{e}>\frac{1-\frac{\beta_{\|}}{2}}{1+\frac{1}{\nu \mu}}
$$


and $b<0$ if

$$
P_{e}>\frac{1-\beta_{\|}}{1+\frac{\mu}{\nu}}
$$

For a low $\beta_{\|}<1$

$$
\frac{1-\frac{\beta_{\|}}{2}}{1+\frac{1}{\nu \mu}}>\frac{1-\beta_{\|}}{1+\frac{\mu}{\nu}},
$$

leading to the necessary condition $b<0$, also found in Ref. 4, Eq. (50). Looking to the solutions of Eq. (3) we can easily observe that this condition $b<0$ is also sufficient to have at least one solution positive

$$
y=\frac{-a+\sqrt{a^{2}-4 b}}{2}>0,
$$

that yields, explicitly,

$$
\begin{aligned}
x_{0}^{2}= & \frac{1}{2}\left[P_{e}\left(1+\frac{1}{\nu \mu}\right)+\frac{\beta_{\|}}{2}-1\right] \\
& +\frac{1}{2}\left[\left(\frac{\beta_{\|}}{2}-1\right)^{2}+P_{e}^{2}\left(1+\frac{1}{\nu \mu}\right)^{2}-2 P_{e}\left(1-\frac{\beta_{\|}}{2}\right)\right. \\
& \left.+\frac{2 P_{e}}{\nu \mu}\left(1+\frac{\beta_{\|}}{2}\right)\right]^{0.5} .
\end{aligned}
$$

Otherwise, for a high $\beta_{\|}>1, b<0$ is satisfied for any value of $P_{e}$, and the same solution (9) remains positive.

\section{B. Interlude: condition $b<0$}

Here we analyze in detail the necessary condition $b<0$ for particular cases, when only the electrons are counterstreaming and ions are stationary (forming just a neutralizing background), and for neutral beams when both the electrons and ions have the same streaming velocities.

\section{Counterstreams of electrons}

When ions are at rest, $V_{i}=0$, then $\nu \rightarrow \infty$, and the instability condition (7) becomes (also see Eq. (50) from Ref. 4)

$$
P_{e}>1-\beta_{\|}
$$

Using the explicit form in Eq. (2), the instability condition (11) requires $V_{e}^{2}>\left(1-\beta_{\|}\right) c^{2} \Omega_{e}^{2} / \omega_{p, e}^{2}$ or

$$
\beta_{\|}>\frac{1}{1+\frac{V_{e}^{2}}{u_{e, \|}^{2}}}
$$

which is less constrained than the condition derived in Ref. 9

$$
\beta_{\|}>\frac{2}{1+\frac{2 V_{e}^{2}}{u_{e, \|}^{2}}}>\frac{1}{1+\frac{V_{e}^{2}}{u_{e, \|}^{2}}}
$$

For cold beams we recover the same condition derived in Ref. $14 V_{e}>c \Omega_{e} / \omega_{p, e}$.

\section{Neutral counterstreams}

When both the electrons and ions are streaming with the same velocity $V_{e}=V_{p}=V$, implying $\nu=1$, the same condition (7) becomes

$$
P_{e}>\frac{1-\beta_{\|}}{1+\mu / \nu}=\frac{1-\beta_{\|}}{\mu} .
$$

Writing $P_{e}$ in terms of Alfven speed $V_{A}=c \Omega_{p} / \omega_{p, p}$

$$
P_{e} \equiv \frac{\omega_{p, e}^{2} V_{e}^{2}}{\Omega_{e}^{2} c^{2}}=\frac{V_{e}^{2}}{\mu V_{A}^{2}}
$$

implies in condition (14)

$$
V>V_{A}\left(1-\beta_{\|}\right)^{1 / 2}
$$

which is the same with condition (7) from Ref. 11. For cold beams we find necessary $V>V_{A}$, the same condition derived in Ref. 15. Since $V_{A}=c \Omega_{p} / \omega_{p, p}=$ $c\left(m_{e} / m_{p}\right)^{0.5}\left(\Omega_{e} / \omega_{p, e}\right)<c \Omega_{e} / \omega_{p, e}$, it follows that a system with counterstreams of protons (ions) is much more susceptible to the instability than one with only counterstreams of electrons.

\section{The instability condition}

With $x_{0}$ derived in Eq. (10) the marginal instability condition is readily found from Eq. (34) in Ref. [4]

$$
A<W\left(x_{0}\right)+\frac{W\left(\mu x_{0}\right)}{\mu}+\frac{2 P_{e}}{\beta_{\|}}\left[W\left(x_{0}\right)+\frac{W\left(\mu x_{0}\right)}{\nu}\right]-\frac{2 x_{0}}{\beta_{\|}},
$$

where $W(z)=1-e^{-z} I_{0}(z)$. We use this condition to derive the marginal stability against the $\mathrm{O}$-mode instability. This condition is displayed with solid lines in Figs. 2 and 3 for a number of relevant cases. The O-mode must be unstable in the gray shading below the solid lines. In Fig. 3 counterstreams are chosen to be neutral, i.e., with $V_{e}=V_{p}$ (case I in Fig. 1), while in Fig. 2 the electron streams are faster than ions $\left(V_{e}>V_{p}\right.$, i.e., case II in Fig. 1) with $\nu=10$.

For the third case, when ions are stationary and only electrons are counterstreaming (case III in Fig. 1), the parameter $\nu \rightarrow \infty$ becomes very large, reducing the expression in Eq. (10)

$$
x_{0}^{2}=P_{e}+\frac{\beta_{\|}}{2}-1,
$$

and the instability condition from Eq. (17)

$$
A<W\left(x_{0}\right)+\frac{W\left(\mu x_{0}\right)}{\mu}+\frac{2}{\beta_{\|}}\left[P_{e} W\left(x_{0}\right)-x_{0}\right] .
$$

Notice that by comparison to Ref. 2, where the instability condition is derived by neglecting the ion effects, here 
FIG. 2: Marginal instability for the OMI from Eq. (17) (solid line), and for the TSI from Eq. (26) (dashed line), when $\nu=V_{e}^{2} / V_{i}^{2}=10$. The OMI can develop only in the darkest shading where streaming velocity is below the threshold required for the onset of TSI.

FIG. 3: Marginal instability for the OMI from Eq. (17) (solid line), and for the TSI from Eq. (26) (dashed line) for neutral counterstreams, i.e., $V_{e}=V_{i}=V(\nu=1)$. The OMI can develop only in the darkest shading where streaming velocity is below the threshold required for the onset of TSI.

the ion nonstreaming effects are still present in the rightside (second term) of inequality (19). This new form in Eq. (19) is used in the present paper to derive the marginal stability of the electron counterstreams, which is displayed with solid lines in Fig. 4.

\section{INTERPLAY WITH THE ELECTROSTATIC TWO-STREAM INSTABILITY}

Counterstreaming plasmas are also subject to the electrostatic streaming instabilities, i.e., electron-electron $(e-e)$, electron-proton $(e-p)$, and proton-proton $(p-p)$ instabilities, with a maximum growth in the streaming direction. Of these, the most efficient (or faster) are the instabilities driven by electrons, i.e., $e-e$ or $e-p$ twostream instabilities $^{12,13}$. Notice that no acoustic mode can be excited if the plasma populations and components are isothermal $\left(T_{e} \sim T_{p}\right)$. The two-stream instability is also faster than the O-mode instability ${ }^{10,11}$, except for streaming velocity very near or below the threshold for the onset of the two-stream instability. The electrostatic instability is only inhibited by the thermal spread of plasma particles in the streaming direction (i.e., the parallel temperature), and it is therefore expected to become even more competitive against the O-mode instability in the low $\beta<1$ regime, where either the two-stream instability is enhanced by the low temperature, or the $\mathrm{O}$-mode instability is inhibited by the intense magnetic field ${ }^{1,9-11}$.

For that reason, the existence of the O-mode instability can be well established only below the marginal condition for the two-stream instability. Here we propose to delimitate these regimes based on the results in Ref. [13], where the marginal condition has been derived systematically for different two-stream instabilities. The symmetry conditions imposed in our present study for the counterstreaming plasmas (enables decoupling of the O-mode from the X-mode) along with $T_{e} \simeq T_{p}$ (ubiquitous in space plasmas) lead to a reduced number of two-stream instabilities specified in Table I. Relevant for us here are only the plasma counterstreams $a-b$ of the same species $a=b=e, p$ or different species $a=e, b=p$, but satisfying

$$
\Lambda \equiv\left(\frac{\epsilon_{a} T_{b}}{\epsilon_{b} T_{a}}\right)^{1 / 2}=1,
$$

(since $\epsilon_{a}=\epsilon_{b}=1 / 2$, and $T_{a}=T_{b}$, see previous section).
In this case the marginal condition of stability for two generic counterstreams $a-b$ is ${ }^{13}$

$$
\frac{\left|\vec{V}_{b}-\vec{V}_{a}\right|}{u_{b, \|}} \leqslant 0.92\left(1+\frac{\omega_{p, b}}{\omega_{p, a}}\right),
$$

We analyze this condition for each type of two-stream instability found relevant in Table I, and then compare them to derive the lowest threshold condition.

\section{A. Electron-electron streaming instability}

In this case $a=b=e$, the relative velocity reads $\mid \vec{V}_{b}-$ $\vec{V}_{a} \mid=2 V_{e}$ and the marginal condition (21) becomes $^{12}$

$$
V_{e} \leqslant 0.92 u_{e, \|}
$$

Now, to adapt within a $A \propto \beta_{\|}^{-1}$-dependence (temperature anisotropy vs. inverse plasma beta) we use definition (2), and express condition (22) in terms of the (electron) streaming parameter $P_{e}$ and the parallel plasma beta

$$
P_{e} \leqslant 0.85 \beta_{\|}
$$

\section{B. Proton-proton streaming instability}

If the instability is driven only by protons, i.e., $a=b=$ $p$, the marginal condition (21) provides a lower threshold for the streaming velocity of protons (also discussed by Stringer $^{12}$ )

$$
V_{p} \leqslant 0.92 u_{p, \|}
$$

since $u_{p, \|}=u_{e, \|} / \mu^{1 / 2}<u_{e, \|}\left(\mu=m_{p} / m_{e}=1836\right)$. The first two cases in Fig. 1 include conditions when the proton streaming velocity is high enough to drive the two-stream $p-p$ instability. However, developing of this instability (with a growth rate of the order of proton plasma frequency) is not realistic since the O-mode instability (with a growth rate of the order of electron gyrofrequency) is expected to be much faster.

\section{Electron-proton streaming instability}

In this case a Buneman-like instability is induced $^{12,16,17}$, and the lowest threshold is found 
FIG. 4: Marginal instability for the OMI, from Eq. (19) (solid line), and for the TSI from Eq. (23) (dashed line), driven only by the counterstreams of electrons (stationary ions, $V_{i}=0$ ). The OMI can develop only in the darkest shading where the streaming velocity is below the threshold required for the onset of TSI.

TABLE I: Types of electrostatic two-stream instabilities relevant for our cases I, II, and III, and the lowest marginal condition of stability.

\begin{tabular}{llll}
\hline \hline & $\nu$ & Instabilities & Marginal condition \\
\hline I. & $\nu=1$ & $e-e, e-p, p-p$ & Eq. (27) \\
II. & $\nu<\mu$ & $e-e, e-p, p-p$ & Eq. (26) \\
& $\nu>\mu$ & $e-e, e-p, p-p$ & Eq. (23) \\
III. & $\nu \rightarrow \infty$ & $e-e, e-p$ & Eq. (23) \\
\hline \hline
\end{tabular}

from the same condition (21) if we consider the case $a=p$ and $b=e$

$$
V_{e} \leqslant 0.92 u_{e, \|} \frac{1+\frac{1}{\mu^{1 / 2}}}{1 \pm \frac{1}{\nu^{1 / 2}}}
$$

Furthermore, we can distinguish between two growing modes distinctively assigned to " \pm " in the denominator. Thus, the instability can be driven by electrons and protons streaming either in the same direction, when the relative drifting speed is only $\left|\vec{V}_{e}-\vec{V}_{p}\right|=V_{e}-V_{p}$, or in opposite directions, when the relative drifting speed is higher $\left|\vec{V}_{e}-\vec{V}_{p}\right|=V_{e}+V_{p}$ and determines a lower threshold, asigned to "+" in the denominator in Eq. (25). In terms of the streaming parameter $P_{e}$ and the parallel plasma beta this condition reads

$$
P_{e} \leqslant 0.85 \beta_{\|}\left(\frac{1+\frac{1}{\mu^{1 / 2}}}{1+\frac{1}{\nu^{1 / 2}}}\right)^{2}
$$

Now, comparing conditions (23) and (26), we find that the lowest threshold is defined by (23) only if $\nu>\mu=$ 1836, condition well satisfied when ions are almost stationary, e.g., the limit case III. Indeed, in the limit case III, the ions are stationary $\nu \rightarrow \infty$, and the two-stream $e-e$ instability presents the lowest marginal condition given by (23). In the other limit of neutral counterstreams, when the electrons and ions move with the same streaming velocity, $\nu=1$ and the lowest marginal condition is that against the electron-proton instability reduced to

$$
P_{e} \leqslant 0.21 \beta_{\|}\left(1+\frac{1}{\mu^{1 / 2}}\right)^{2} \simeq 0.22 \beta_{\|}<0.92 \beta_{\|} .
$$

The lowest marginal conditions of stability against the electrostatic two-stream instabilities are summarized in Table I, indicating three different cases, which are not necessarily related to our first classification, i.e., cases I, II and III.
Thus, a new distinction can be made function of the parameter $\nu$ as it takes values less or higher than the protonelectron mass ratio $\mu$. If $\nu<\mu$ is satisfied the lowest marginal condition against any electrostatic instability is given by (26). For an arbitrary value $\nu=10$ (case also studied in Ref.[4]) this condition becomes $P_{e} \leqslant 0.51 \beta_{\|}$, and it is displayed with dashed lines in Fig. 2. For the limit case when $\nu=1$, the same marginal condition simplifies to (27), which is displayed with dashed lines in Fig. 3. In the opposite case, when $\nu>\mu$ is satisfied, including the limit case $\nu \rightarrow \infty$ of stationary ions, the lowest marginal condition against any electrostatic instability is given by (23). This condition is displayed with dashed lines in Fig. 4.

The regimes where only the O-mode instability can operate are always found in the right-hand side of these dashed lines, i.e., the lighter gray shading. The superposition with the conditions for O-mode instability indicates for the existence of this instability only the darkest shading regions. In the high beta $\left(\beta_{\|}>1\right)$ plasmas the existence of the O-mode instability is not significantly affected, unless the streams are very energetic $\left(P_{e}>1\right)$. But the existence of this instability is drastically restrained by the interplay with the two-stream instability in the low beta $\left(\beta_{\|}<1\right)$ regimes.

\section{DISCUSSION AND CONCLUSIONS}

We have studied the marginal conditions for the Omode instability by contrast to those of the electrostatic instabilities. In the process of relaxation of the counterstreaming plasmas the electrostatic two-stream instabilities (with a growth rate of the order of electron plasma frequency) are usually much faster than the O-mode instability (with a growth rate of the order of electron gyrofrequency). The existence of the O-mode can therefore be established only for streaming velocities below the threshold of the two-stream instabilities.

Our present analysis is based on accurate analytical expressions of the marginal conditions of instability provided in Ref. 4 for the O-mode instability, and in Ref. 13 for the two-stream instabilities. The refined analysis in Secs. II and III aims to assess the robustness of these analytical expressions by the agreements found with particular cases studied before, e.g., stationary ions, neutral streams, $e-e$ or $e-p$ counterstreams. Despite the limitations imposed by the symmetry of the counterstreams, this seems to be the most convenient way to study the O-mode (decoupled from other plasma modes) and make its properties more transparent. Presently there is an increased interest for the O-mode instability, especially for understanding its activity in the low-beta plasmas 
$(\beta<1)$ and for temperature anisotropies $A=T_{\perp} / T_{\|}$ even larger than unity. As a mechanism of limitation of the kinetic anisotropies, this instability could provide a plausible explanation for the low-beta boundaries of stable plasma configurations observed in the solar wind and terrestrial magnetosphere.

Our investigation on the competition with the twostream instabilities reveals that the parameter range of the O-mode instability is significantly restrained, especially in the low-beta plasmas. Thus the low-beta regimes where only the O-mode instability can operate are significantly restrained by a minimum cutoff, given by

$$
\beta_{\|}>\beta_{\|, c} \equiv \frac{P_{e}}{0.85}\left(\frac{1+\frac{1}{\nu^{1 / 2}}}{1+\frac{1}{\mu^{1 / 2}}}\right)^{2}
$$

if $\nu<\mu=1836$ is satisfied, see Figs. 2 and 3, or by

$$
\beta_{\|}>\beta_{\|, c} \equiv \frac{P_{e}}{0.85}
$$

in all the other cases, see Fig. 4. The activity of this instability in the high-beta plasmas can be also constrained by the two-stream instability, if $P_{e}$ is large enough, see bottom panels in Figs. 2 and 3.

Moreover, the existence of the O-mode instability becomes limited only to sufficiently small $A=T_{\perp} / T_{\|}$, less than a maximum value $A_{m}$ given by Eqs. (17) and (10) at the plasma beta cutoff $\left(\beta_{\|, c}\right)$ derived above, i.e.,

$A_{m}=W\left(x_{c}\right)+\frac{W\left(\mu x_{c}\right)}{\mu}+\frac{2 P_{e}}{\beta_{\|, c}}\left[W\left(x_{c}\right)+\frac{W\left(\mu x_{c}\right)}{\nu}\right]-\frac{2 x_{c}}{\beta_{\|, c}}$

with

$$
\begin{aligned}
x_{c}^{2}= & \frac{1}{2}\left[P_{e}\left(1+\frac{1}{\nu \mu}\right)+\frac{\beta_{\|, c}}{2}-1\right] \\
& +\frac{1}{2}\left[\left(\frac{\beta_{\|, c}}{2}-1\right)^{2}+P_{e}^{2}\left(1+\frac{1}{\nu \mu}\right)^{2}\right. \\
& \left.-2 P_{e}\left(1-\frac{\beta_{\|, c}}{2}\right)+\frac{2 P_{e}}{\nu \mu}\left(1+\frac{\beta_{\|, c}}{2}\right)\right]^{0.5} .
\end{aligned}
$$

Estimations can be made if we, for instance, take a few examples of a less particular case when $\nu<\mu$, like the ones displayed in Fig. $2(\nu=10)$. In this case $\beta_{\|, c} \simeq 1.96 P_{e}$ and $x_{c}^{2}=0.5\left(3.92 P_{e}^{2}-3.96 P_{e}+1\right)^{0.5}+$ $0.99 P_{e}-0.5 \simeq 2 P_{e}-1$, and the limit values $A_{m}$ calculated with Eq. (30) are listed in Table II for different values of the parameter $P_{e}$. Notice that values larger than unity $A_{m}>1$ for the temperature anisotropy are admitted, but only for sufficiently large electron streaming velocities corresponding to $P_{e}>1$, and only for sufficiently large values of the plasma beta parameter $\beta_{\|}>1$.
To conclude, the conditions where the O-mode instability can operate efficiently are markedly constrained by the electrostatic instabilities in both the low $\beta_{\|}<1$ and large $\beta_{\|}>1$ plasmas. Thus the existence of the O-mode

TABLE II: Limit (maximum) values of the temperature anisotropy $\left(A_{m}\right)$ given by Eq. (30) for the cases displayed in Fig. 2.

\begin{tabular}{llllll}
\hline \hline$P_{e}$ & 0.05 & 0.1 & 0.5 & 1.0 & 5.0 \\
\hline$\beta_{\|, c}$ & 0.098 & 0.19 & 0.98 & 1.96 & 9.8 \\
$x_{c}\left(\times 10^{-2}\right)$ & 0.17 & 0.26 & 4.72 & 99.0 & 298 \\
$A_{m}$ & 0.047 & 0.062 & 0.094 & 0.170 & 1.02 \\
\hline \hline
\end{tabular}

instability becomes possible only for sufficiently high values of the plasma beta parameter in parallel direction $\beta_{\|} \geqslant \beta_{\|, c}$. This restriction is particularly important for the low-beta plasmas, since the existence of this instability was claimed in the previous studies for any small value (without limit) of the parallel plasma beta $\beta_{\|} \rightarrow 0$. The instability of the O-mode can develop in the lowbeta plasmas but only for $\nu<\mu$ and a streaming energy density less than the magnetic energy density $\left(P_{e}<1\right)$. Further comparison is now possible with the solar wind streaming conditions to establish whether this instability can explain the observed limits of the anisotropy or not. For a supra-unitary anisotropy $A=T_{\perp} / T_{\|}>1$, the stimulation of this instability by the energetic streams seems to be impossible (or unrealistic) in the low-beta plasmas. These situations appear to be resolved by a more realistic dissipation of the streaming free energy by the electrostatic instabilities.

\section{Acknowledgments}

The authors acknowledge support from the Katholieke Universiteit Leuven, Grant nr. SF/12/003, and from the Ruhr-Universität Bochum, the Deutsche Forschungsgemeinschaft (DFG), Grants Schl 201/25-1 and SH 21/32 . These results were obtained in the framework of the projects GOA/2015-014 (KU Leuven), G.0729.11 (FWOVlaanderen) and C 90347 (ESA Prodex 9). The research leading to these results has also received funding from the European Commission's Seventh Framework Programme (FP7/2007-2013) under the grant agreements SOLSPANET (project n 269299, www.solspanet.eu) and eHeroes (project n 284461, www.eheroes.eu).
1. A. Stockem, I. Lerche and R. Schlickeiser, Astrophys. J. 651, 584 (2006).
2. D. Ibscher, M. Lazar, M. J. Michno and R. Schlickeiser, 
Phys. Plasmas 20, 012103 (2013).

3. F. Hadi, M. F. Bashir, A. Qamar, P. Yoon and R. Schlickeiser, Phys. Plasmas 21, 052111 (2014).

4. R. Schlickeiser and P. Yoon, Phys. Plasmas 21, 072119 (2014).

5. D. Ibscher, M. Lazar, and R. Schlickeiser, Phys. Plasmas 19, 072116 (2012)

6. S. Hamasaki, Phys. Fluids 11, 2724 (1968).

7. R. C. Davidson and C. S. Wu, Phys. Fluids 13, 1407 (1970).

8. M. Lazar, S. Poedts, R. Schlickeiser and D. Ibscher, Solar Phys. 289, 369 (2013).
9. M. Bornatici and K. F. Lee, Phys. Fluids 13, 3007 (1970).

10. K. F. Lee and J. C. Armstrong, Phys. Rev. A 4, 2087 (1971).

11. J. D. Gaffey, W. B. Thompson and C. S. Liu, J. Plasma Phys. 7, 189 (1972).

12. T. E. Stringer, Plasma Phys. 6, 267 (1964).

13. V. Lapuerta and E. Ahedo, Phys. Plasmas 9, 1513 (2002).

14. K. F. Lee, Phys. Rev. 181, 447 (1969).

15. K. F. Lee, J. Appl. Phys. 41, 3045 (1970).

16. O. Buneman, Phys. Rev. 115, 503 (1959).

17. E. Ahedo and V. Lapuerta, Phys. Plasmas 8, 3873 (2001). 


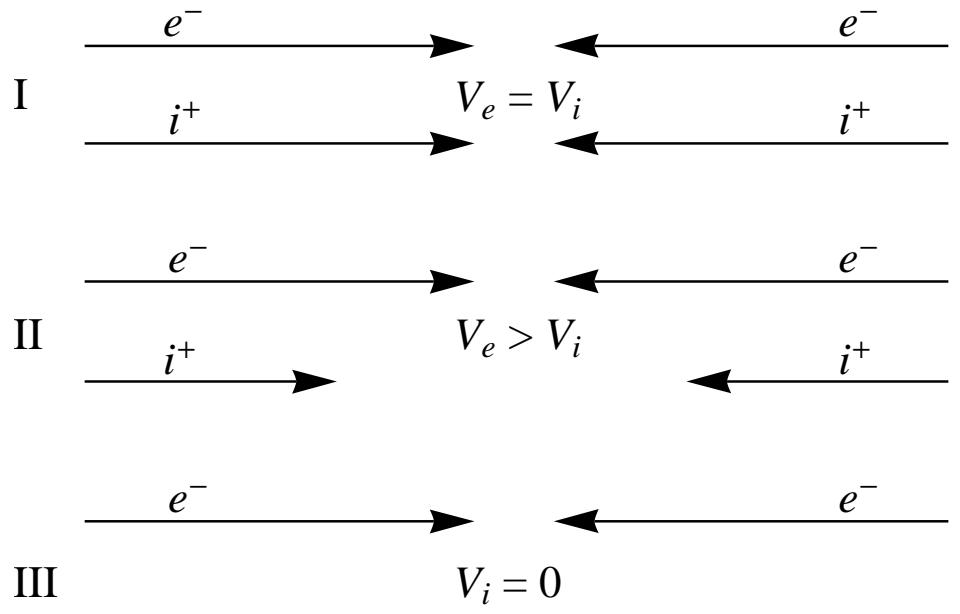




$$
v=10 ; P_{e}=0.01
$$

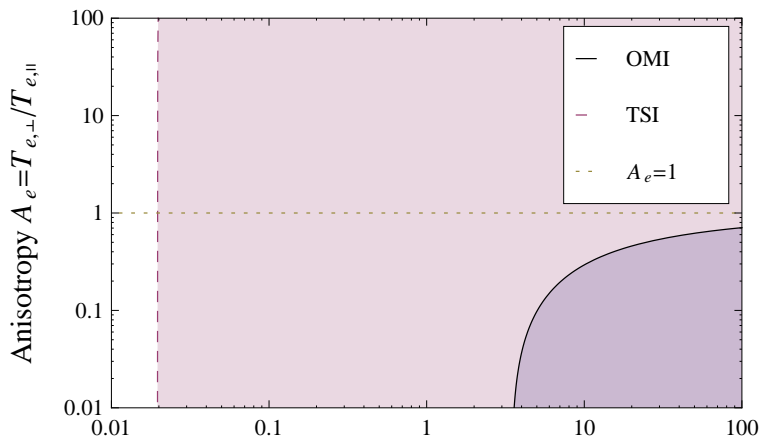

parallel beta $\beta_{\text {॥ }}$ 


$$
v=10 ; P_{e}=0.05
$$

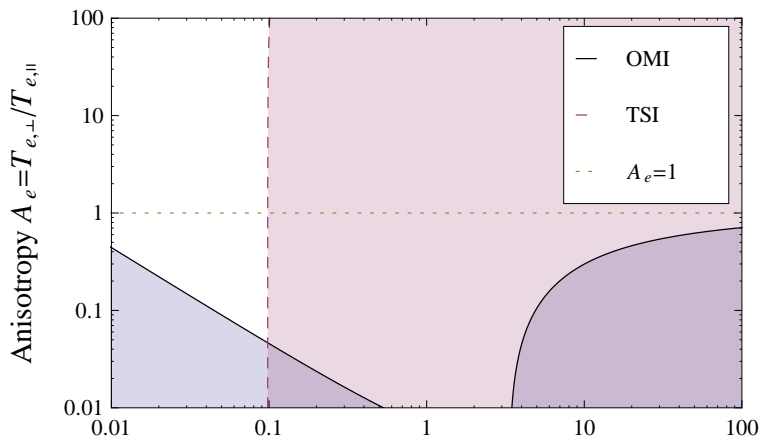

parallel beta $\beta_{\text {॥ }}$ 


$$
v=10 ; P_{e}=0.1
$$

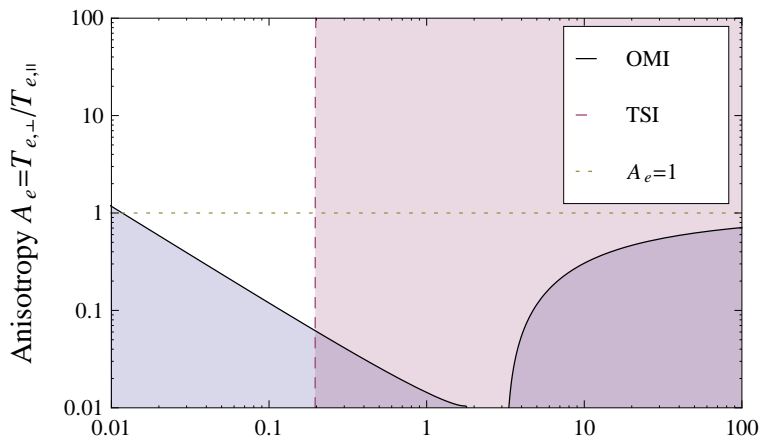

parallel beta $\beta_{\text {॥ }}$ 


$$
v=10 ; P_{e}=0.5
$$

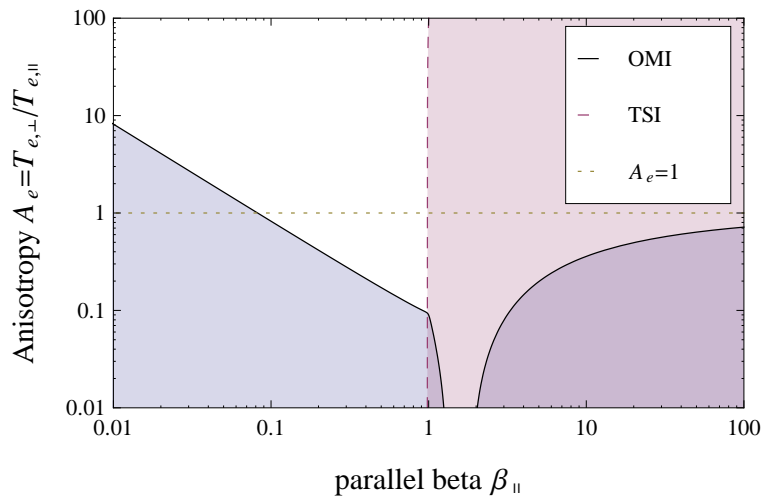




$$
v=10 ; P_{e}=1
$$

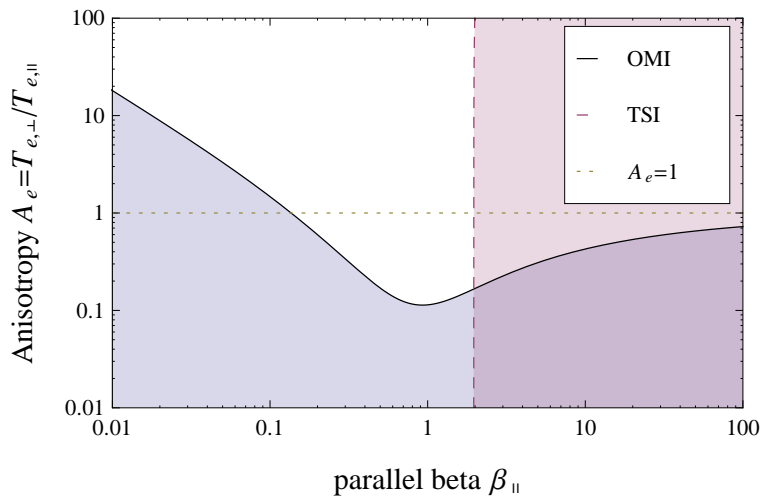




$$
v=1 ; P_{e}=0.01
$$

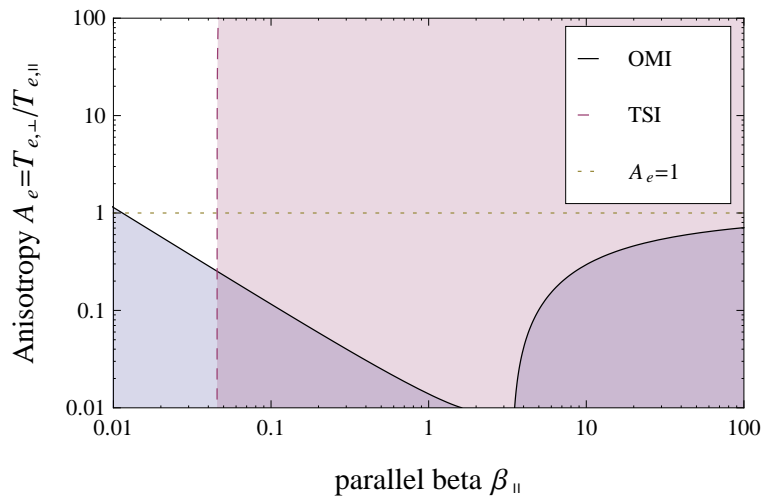




$$
v=1 ; P_{e}=0.05
$$

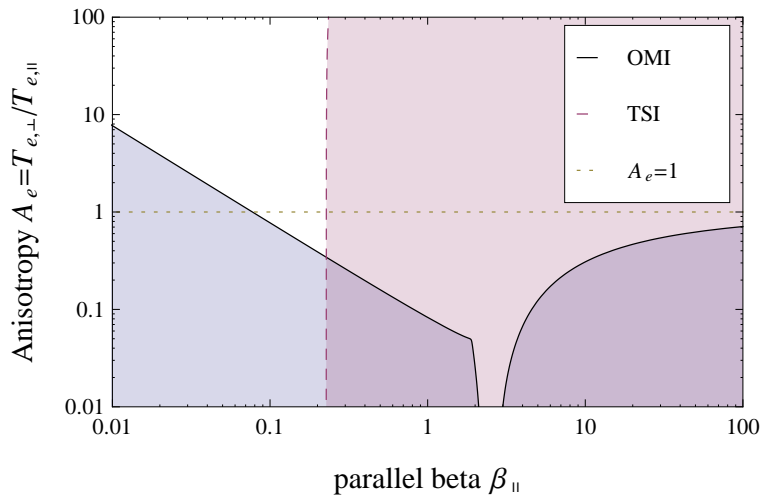




$$
v=1 ; P_{e}=0.1
$$

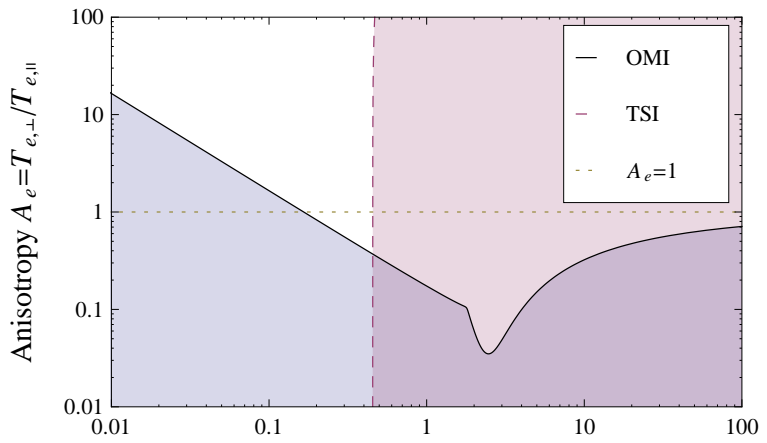

parallel beta $\beta_{\text {॥ }}$ 


$$
v=1 ; P_{e}=0.5
$$

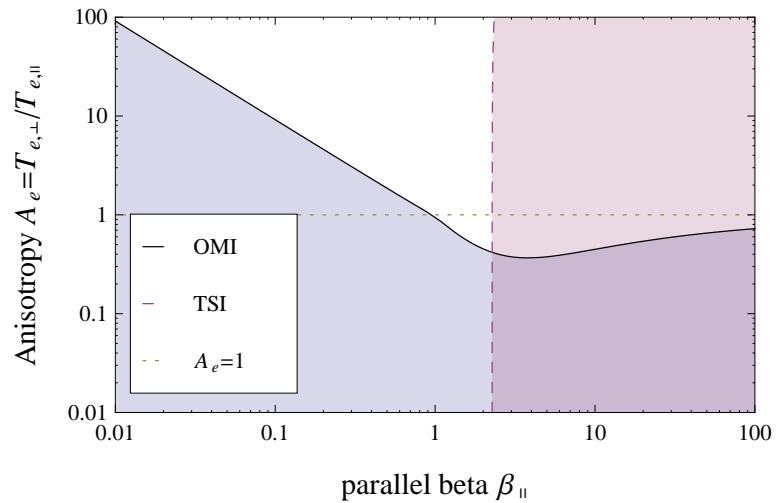




$$
v=1 ; P_{e}=1
$$

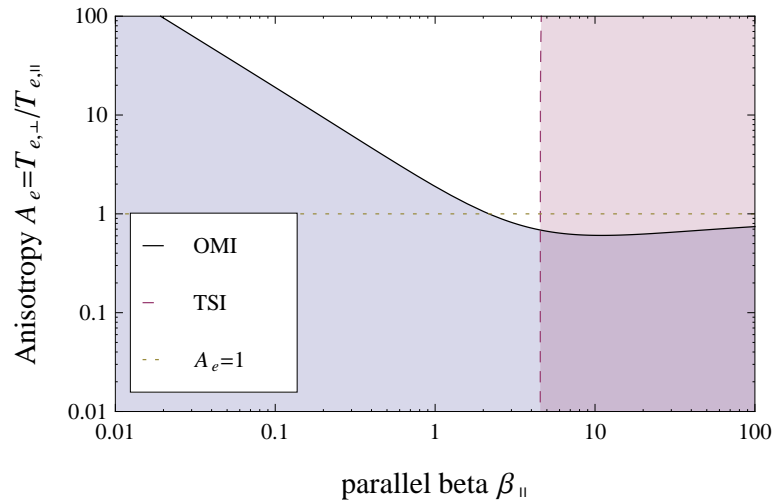




$$
v=1 ; P_{e}=5
$$

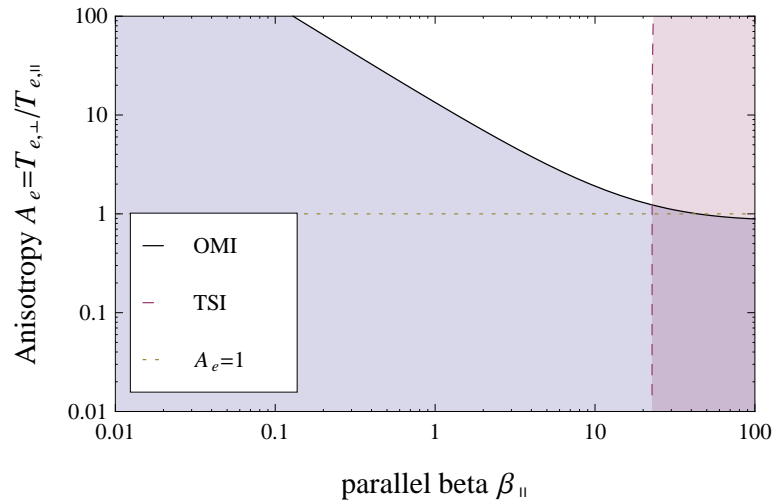




$$
v \rightarrow \infty ; P_{e}=0.1
$$

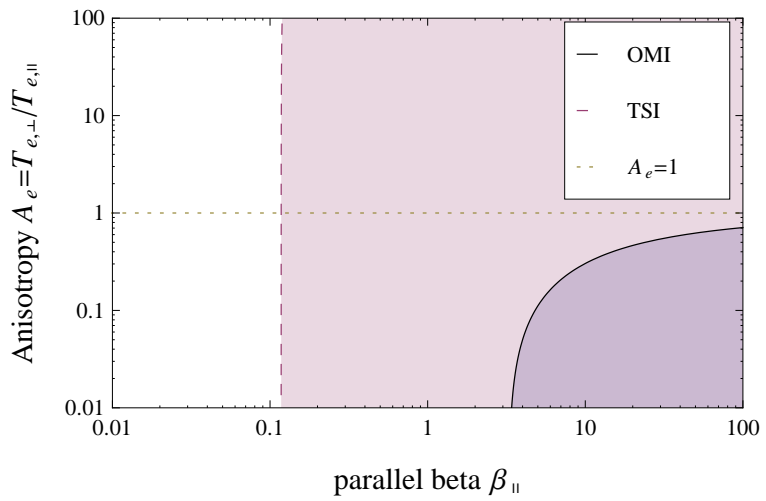




$$
v \rightarrow \infty ; P_{e}=1
$$

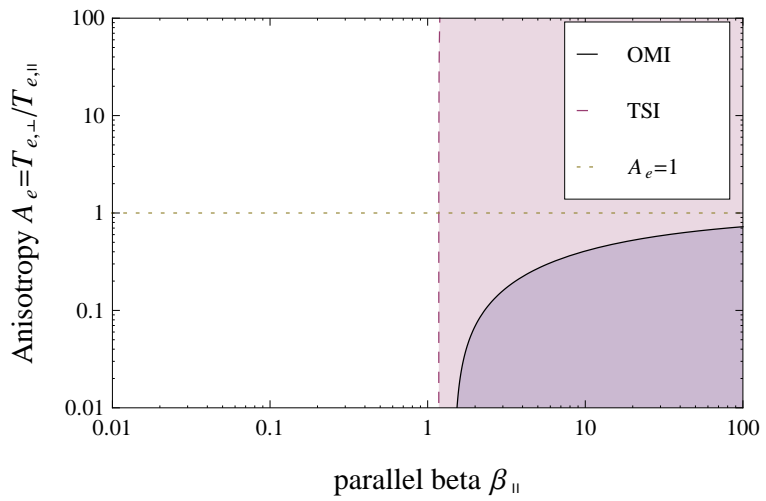


$\nu \rightarrow \infty ; P_{e}=1.73$

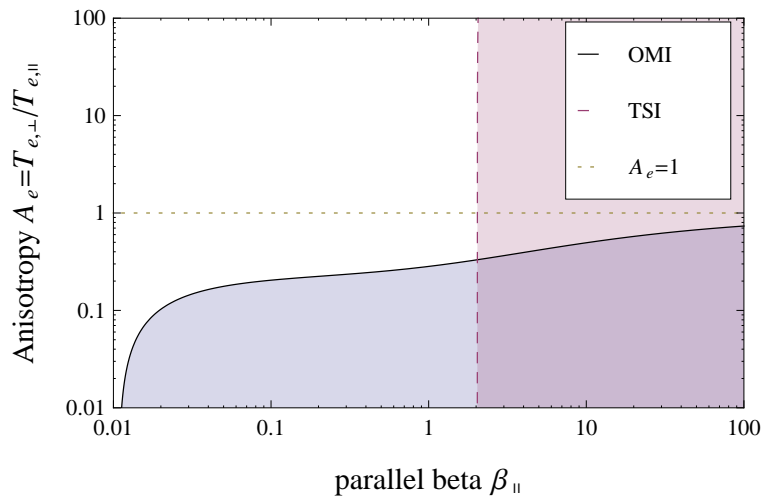


$v \rightarrow \infty ; P_{e}=1.74$

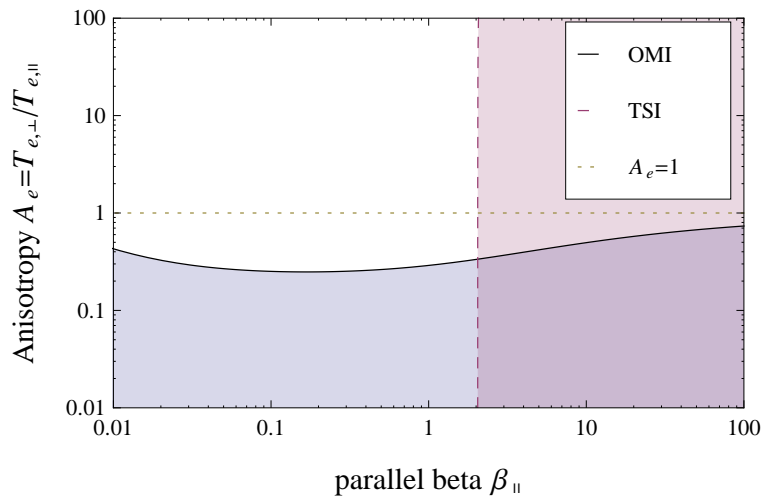




$$
v \rightarrow \infty ; P_{e}=1.8
$$

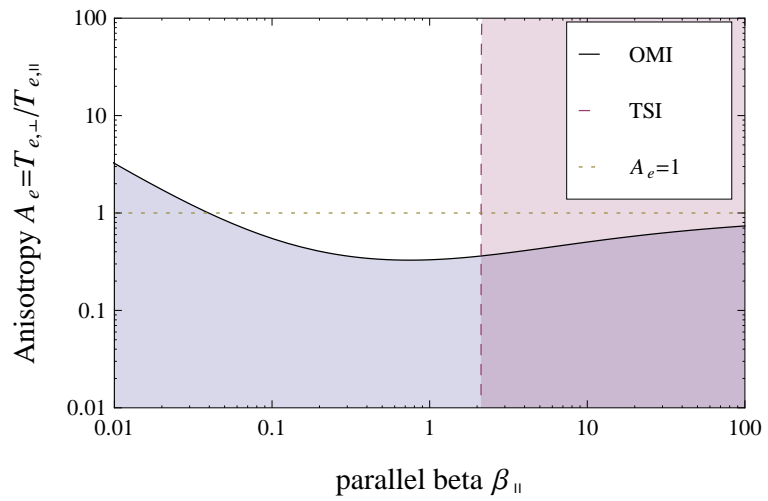


